\title{
Tissue-specific splicing of a ubiquitously expressed transcription factor is essential for muscle differentiation
}

\author{
Soji Sebastian, ${ }^{1}$ Hervé Faralli, ${ }^{1,4}$ Zizhen Yao, ${ }^{2,4}$ Patricia Rakopoulos, ${ }^{1,3}$ Carmen Palii, ${ }^{1}$ Yi Cao, ${ }^{2}$ \\ Kulwant Singh, ${ }^{1}$ Qi-Cai Liu, ${ }^{1}$ Alphonse Chu, ${ }^{1}$ Arif Aziz, ${ }^{1}$ Marjorie Brand, ${ }^{1,3}$ Stephen J. Tapscott, ${ }^{2}$ \\ and F. Jeffrey Dilworth ${ }^{1,3,5}$ \\ ${ }^{1}$ Sprott Center for Stem Cell Research, Ottawa Hospital Research Institute, Ottawa, Ontario K1H 8L6, Canada, ${ }^{2}$ Human Biology \\ Division, Fred Hutchinson Cancer Research Center, Seattle, Washington 98109, USA; ${ }^{3}$ Department of Cellular and Molecular \\ Medicine, University of Ottawa, Ontario K1H 8L6, Canada
}

\begin{abstract}
Alternate splicing contributes extensively to cellular complexity by generating protein isoforms with divergent functions. However, the role of alternate isoforms in development remains poorly understood. Mef2 transcription factors are essential transducers of cell signaling that modulate differentiation of many cell types. Among Mef2 family members, Mef2D is unique, as it undergoes tissue-specific splicing to generate a muscle-specific isoform. Since the ubiquitously expressed (Mef2D 1 ) and muscle-specific (Mef2D $\alpha 2$ ) isoforms of Mef2D are both expressed in muscle, we examined the relative contribution of each Mef2D isoform to differentiation. Using both in vitro and in vivo models, we demonstrate that Mef2D isoforms act antagonistically to modulate differentiation. While chromatin immunoprecipitation (ChIP) sequencing analysis shows that the Mef2D isoforms bind an overlapping set of genes, only Mef2Da2 activates late muscle transcription. Mechanistically, the differential ability of Mef2D isoforms to activate transcription depends on their susceptibility to phosphorylation by protein kinase A (PKA). Phosphorylation of Mef2Da1 by PKA provokes its association with corepressors. Conversely, exon switching allows Mef2D 2 to escape this inhibitory phosphorylation, permitting recruitment of Ash2L for transactivation of muscle genes. Thus, our results reveal a novel mechanism in which a tissue-specific alternate splicing event has evolved that permits a ubiquitously expressed transcription factor to escape inhibitory signaling for temporal regulation of gene expression.
\end{abstract}

[Keywords: alternative splicing; Mef2; PKA signaling; gene expression; HDAC; Ash2L]

Supplemental material is available for this article.

Received February 4, 2013; revised version accepted April 26, 2013.

Genome-wide studies have highlighted the capacity of cells to exploit the 20,000 genes of the human genome to produce a far more complex proteome through the generation of alternate transcripts (The ENCODE Project Consortium et al. 2012). A major contributor to this cellular complexity is alternative splicing of exons, which has been proposed to occur universally in multiexon genes (Wang et al. 2008). The generation of these alternatively spliced transcripts can occur through several mechanisms, including (1) the inclusion/exclusion of an exon, (2) alternate $3^{\prime}$ or $5^{\prime}$ splice donor/acceptor sites, or (3) the alternate use of mutually exclusive exons (Kalsotra and Cooper 2011). In the case of mutually exclusive exons, the con-

\footnotetext{
${ }^{4}$ These authors contributed equally to this work.

${ }^{5}$ Corresponding author

E-mail jdilworth@ohri.ca

Article published online ahead of print. Article and publication date are online at http://www.genesdev.org/cgi/doi/10.1101/gad.215400.113. Freely available online through the Genes \& Development Open Access option.
}

trolled use of alternate exons allows for the expression of functionally distinct protein isoforms that have the potential to perform specific tasks in a temporally or spatially restricted manner. Furthermore, ontology analysis suggests that transcripts incorporating mutually exclusive exons are likely to play a role in tissue-specific functions such as differentiation (Wang et al. 2008). However, our knowledge concerning the role of specific alternative splicing events in regulating differentiation remains limited.

During myogenesis, myoblasts undergo a temporally ordered series of changes in gene expression, giving rise to multinucleated myotubes (Bergstrom et al. 2002). These alterations are extensive, as RNA sequencing (RNA-seq) analysis has shown that 7770 genes change their expression during myotube formation (Trapnell et al. 2010). In addition to these changes in transcript abundance, alternate mRNA processing leads to a switch in the major isoform of $7 \%$ of alternative spliced genes (Trapnell et al. 
2010). This drastic change in the myogenic transcriptome suggests an important role for alternative splicing in establishing the myotube fate. Among the genes that undergo a major isoform switch during myogenesis is the transcription factor Mef2D (Trapnell et al. 2010). Mef2D is a member of the Mef2 family of transcription factors, which act as key mediators of signal-dependent transcription in many different cell types to control developmental processes such as differentiation (Potthoff and Olson 2007). Interestingly, the switch in Mef2D isoforms observed during muscle differentiation is a result of an alternate use of mutually exclusive exons (see Fig. 1) that generates an isoform that is expressed exclusively in skeletal and cardiac muscle (Martin et al. 1994). However, the functional relevance of this muscle-specific isoform of Mef2D to muscle development remains unknown. Here we sought to elucidate the differential roles of the ubiquitously expressed (Mef2D $\alpha 1)$ and muscle-specific (Mef2D 2 2) isoforms of Mef2D in skeletal myogenesis.

\section{Results}

The muscle-specific isoform of Mef2D drives the late stages of myogenesis

Advances in our understanding of the relative roles of Mef2D isoforms in myogenesis have been hampered by the lack of reagents that distinguish between the endogenous proteins. Consequently, we generated antibodies that discriminate between the muscle-specific Mef2D $\alpha 2$ isoform and its ubiquitously expressed counterpart, Mef2D $\alpha 1$ (Fig. 1A). Western blot of recombinant Mef2D proteins demonstrate the specificity of our antibodies (Supplemental Fig. 1). Furthermore, these antibodies recognize the endogenous Mef2D isoforms in differentiating myoblasts using Western blot, immunofluorescence, and immunoprecipitation (Supplemental Fig. 1; data not shown). Importantly, a similar efficiency of immunoprecipitation is observed with both isoform-specific antibodies.

Initially, we characterized the expression of Mef2D isoforms during murine myogenesis. Temporal analysis demonstrates a clearly distinct pattern of expression for the two Mef2D isoforms (Fig. 1B,C). In the case of the ubiquitously expressed Mef2D $\alpha 1$, the protein is present in proliferating myoblasts and continues to accumulate throughout differentiation. In contrast, the muscle-specific Mef2D $\alpha 2$ is absent from proliferating myoblasts and is strongly induced between 48 and $72 \mathrm{~h}$ of differentiation (Fig. 1B,C). This pattern of expression suggests a role for Mef2D $\alpha 2$ in late myogenesis.

To further explore the role of Mef2D isoforms in myogenesis, we created isogenic C2 myoblast lines where the exogenous expression of either Mef2D $\alpha 1$ or Mef2D $\alpha 2$ can be induced upon doxycycline (Dox) treatment (Supplemental Fig. 2A). Interestingly, myoblasts induced to express Mef2D 22 formed multinucleated, myosin heavy chain (MHC)-positive cells more quickly than the control cells (Fig. 2A; Supplemental Fig. 2B), suggesting an accelerated differentiation. In contrast, expression of Mef2D $\alpha 1$ appeared to block myogenesis, as fewer $\mathrm{MHC}^{+}$cells were
A

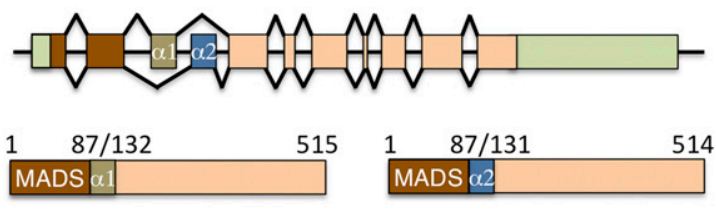

B

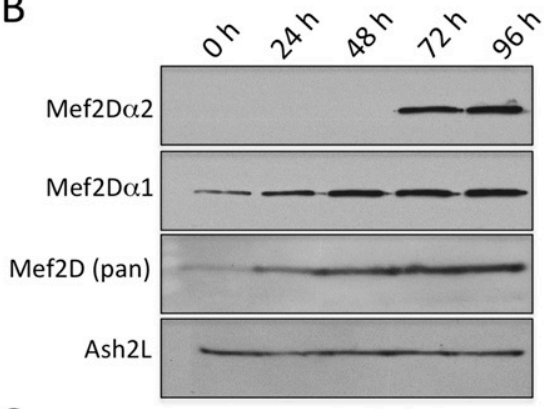

C

$\mathrm{Oh}$

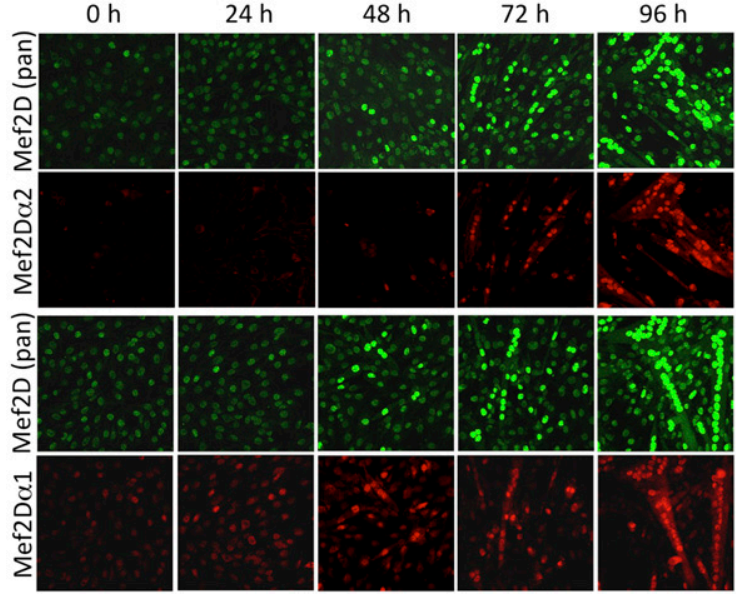

Figure 1. Mef2D undergoes alternative splicing during muscle differentiation to up-regulate the Mef2D $\alpha 2$ isoform. (A) Schematic representation of the intron-exon structure of the Mef2D gene. Alternative incorporation of the mutually exclusive third $(\alpha 1)$ or fourth $(\alpha 2)$ exon results in the generation of an mRNA encoding Mef2D $\alpha 1$ or Mef2D 22 , respectively. The DNA-binding domain of Mef2D proteins is shown in brown (MADS). $(B, C)$ The Mef2D $\alpha 2$ isoform is up-regulated during muscle differentiation. $(B)$ Whole-cell extracts were prepared from C2 cells that were differentiated for various lengths of time $(0-96 \mathrm{~h})$. Western blot was then performed using antibodies specifically recognizing Mef2D $\alpha 1$, Mef2D $\alpha 2$, Mef2D (pan), and Ash2L as indicated. (C) Immunofluorescence analysis was performed on C2 cells that were differentiated for various lengths of time (0-96 h). Slides were stained using antibodies recognizing Mef2D $\alpha 1$, Mef2D 2 , or Mef2D (pan) as indicated.

observed in this population at late stages $(120 \mathrm{~h})$ of differentiation. Having observed opposing effects of Mef2D $\alpha 1$ and Mef2D $\alpha 2$ on differentiation in cultured myoblasts, we next examined whether these disparate roles of Mef2D isoforms are also observed in vivo using a model of mouse muscle regeneration. Indeed, injection of 
A

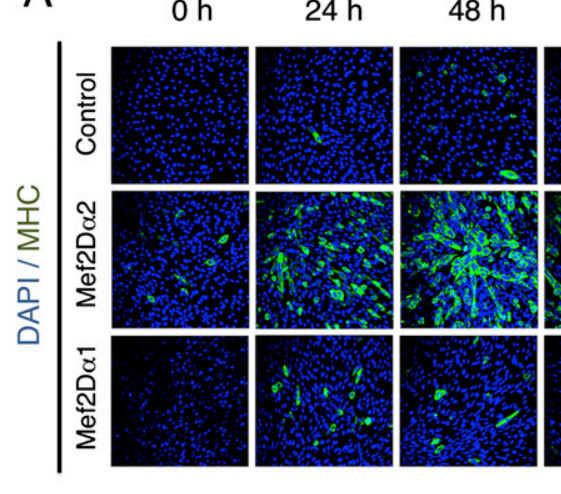

$72 \mathrm{~h}$

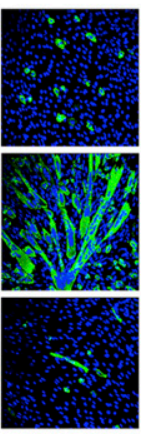

$96 \mathrm{~h}$

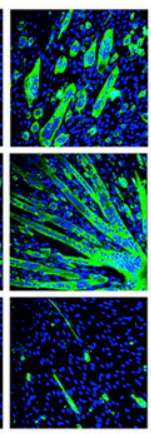

$120 \mathrm{~h}$

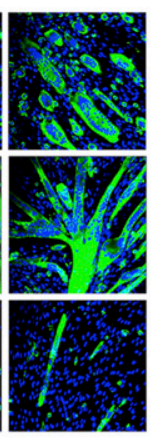

B
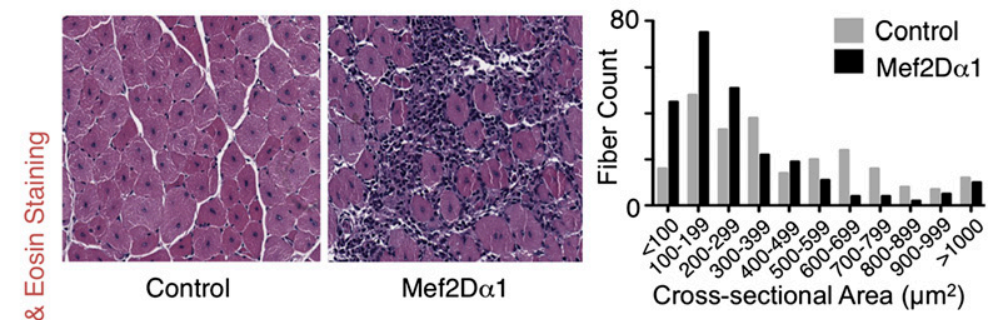

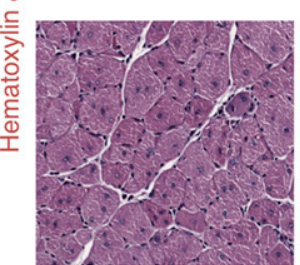

Control

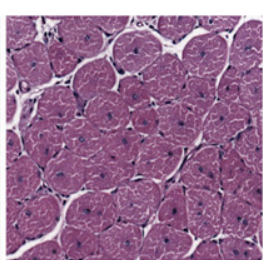

Mef2Da2

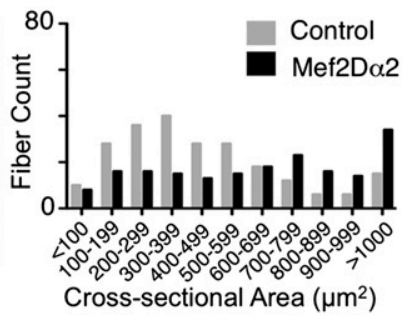

Figure 2. Mef2D $\alpha 1$ and Mef $2 \mathrm{D} \alpha 2$ have opposing effects on muscle differentiation and regeneration. (A) Stable isogenic C2iFRT myoblast lines were induced to express Mef2D $\alpha 1$, Mef2D $\alpha 2$, or control (parental cells). After $24 \mathrm{~h}$ of Dox treatment, cells were incubated in low-serum medium supplemented with Dox to permit differentiation in the presence of exogenous Mef2D isoforms (or control). Immunofluorescence was performed using antibodies recognizing MHC or DAPI as indicated. $(B)$ Cardiotoxin-injured mouse TA muscles were infected with retrovirus expressing Mef2D $\alpha 1$, Mef2D $\alpha 2$, or vector control. After $15 \mathrm{~d}$ of regeneration, cross-sections were stained using hematoxylin and eosin. The cross-sectional area of individual fibers was calculated and plotted. $n=3$. retrovirus expressing Mef2D $\alpha 1$ into cardiotoxin-injured tibialis anterior (TA) muscle resulted in severely impaired regeneration where myofiber repair was delayed (Fig. 2B; Supplemental Fig. 3A), resulting in an accumulation of undifferentiated $\mathrm{MyoD}^{+}$muscle cells in the interstitial space (Supplemental Fig. 4). In contrast, injection of retrovirus expressing Mef2D 22 resulted in improved muscle regeneration with an increased muscle fiber caliber compared with the contralateral control TA muscle (Fig. 2B; Supplemental Fig. 3B). Furthermore, only the injection of Mef2D $\alpha 2$ could mediate the previously described Mef2D-dependent fiber type switching in the TA muscle (Potthoff et al. 2007) from a predominantly fast type MHC-IIb myofiber toward a slower muscle isotype that contained a mixture of MHC-IIb and MHC-IIa myofibers (Supplemental Fig. 5). Thus, Mef2D $\alpha 1$ and Mef2D 22 have antagonistic effects on myogenesis and muscle regeneration.

Examination of Mef2D $\alpha 2$-expressing myoblasts showed that MHC proteins were expressed at the earliest stages of differentiation (0 h) (Fig. 2A). This temporal shift in MHC expression led us to examine whether Mef2D 22 expression would allow for the expression of muscle genes in proliferating myoblasts. To our surprise, RT-qPCR analysis demonstrates that expression of Mef2D 22 , but not Mef2D 1 , can activate Myh3 along with other late myogenic genes (Mylpf and Tnnt1) in proliferating myoblasts (Supplemental Fig. 6). To determine whether the expression of these late markers of myogenesis was due to a precocious differentiation, we examined the ability of Mef2D isoforms to activate expression of Myog and $\mathrm{Ckm}$-two muscle-specific genes normally up-regulated at intermediate stages of myogenesis. In contrast to Myh3, which is strongly up-regulated by ectopic expression of Mef2D $\alpha 2$ in proliferating myoblasts, we observed no change in transcription of $M y o g$ or $C \mathrm{~km}$ upon expression of the muscle-specific isoform (Supplemental Fig. 6). Taken together, our findings suggest that alternate splicing generates the muscle-specific Mef2D $\alpha 2$ isoform to temporally regulate activation of late muscle genes.

We next sought to understand the mechanism underlying the differential ability of Mef2D isoforms to activate late muscle gene expression. Two potential models were envisaged: (1) Mef2D $\alpha 1$ and Mef2D $\alpha 2$ recruit similar coregulatory proteins to an alternate subset of muscle genes or (2) Mef2D $\alpha 1$ and Mef2D $\alpha 2$ bind to a similar set of genomic loci, leading to the recruitment of alternate coregulatory proteins. We therefore explored the contribution of these two models to the functional differences observed between Mef2D isoforms. 
Genome-wide binding shows that Mef2D isoforms bind to a largely overlapping set of genes

We first explored the contribution of altered genomic targeting to the differential ability of Mef2D isoforms to activate transcription. For this, we performed chromatin immunoprecipitation (ChIP) sequencing (ChIP-seq) to examine the genomic distribution of Mef2D $\alpha 1$ and Mef2D $\alpha 2$ in myotubes. Fragmented chromatin was immunoprecipitated using our isoform-specific antibodies to isolate DNA associated with the endogenous Mef2D $\alpha 1$ and Mef2D $\alpha 2$ proteins (Supplemental Figs. 7, 8). Bioinformatic analysis of ChIP-seq data identified 7910 binding sites for Mef2 $\alpha 1$, while the muscle-specific isoform Mef2D $\alpha 2$ bound to 9103 sites that are not present in the control (Fig. 3A; Supplemental Table 1). Comparison of the Mef2D-binding sites using a highly stringent cutoff of $P<10^{-6}$ revealed that $66 \%$ of Mef2D 1 -bound peaks are also bound by Mef2D $\alpha 2$ (Fig. 3A). However, when the stringency is modestly reduced to $P<10^{-4}$, the overlap in binding of Mef2D $\alpha 1$ at Mef2D $\alpha 2$-bound peaks increases to $85 \%$ (data not shown). Consistent with the fact that Mef2D $\alpha 1$ and Mef2D $\alpha 2$ bind to a largely overlapping set of genomic loci, position weight matrices for Mef2D-binding elements observed under these peaks show that both isoforms of the protein recognize a nearly identical consensus sequence of CTAAAAATAG (Fig. 3B). A de novo search for DNA sequences enriched under peaks identified Mef2 and E-box sequences (i.e., MyoD/Myog/Myf5/ MRF4-binding sites) as the two most highly represented DNA elements at both Mef2D $\alpha 1$ and Mef2D $\alpha 2$ genomic targets (data not shown). Interestingly, examination of genomic loci bound preferentially by either Mef2D $\alpha 1$ or Mef2D $\alpha 2$ did not identify Mef2-binding sites as the most highly represented DNA sequences found under these peaks (Supplemental Fig. 9). This finding suggests that the differential binding observed between Mef2D $\alpha 1$ and Mef2D $\alpha 2$ at a small subset of genes is likely due to altered targeting of the transcription factor to DNA through an indirect tethering mechanism.

To assess the importance of overlapping Mef2D $\alpha 1$ and Mef2D $\alpha 2$ genomic binding to myogenesis, we performed gene ontology (GO) analysis of peaks bound by both isoforms. Genes targeted by both Mef2D $\alpha 1$ and Mef2D $\alpha 2$ are strongly associated with GO annotations defining a role in muscle development (Fig. 3C; Supplemental Table 1). Among these genes, we examined Mef2D binding at the HDAC9 gene, which is known to participate in a negative feedback loop with Mef2 proteins (Haberland et al. 2007). At this locus, we observed peaks within the HDAC9 promoter for both Mef2D $\alpha 1$ and Mef2D $\alpha 2$ that overlap with MyoD binding (Fig. 3D). Similarly, we observed that the promoters of Myh3, Titin, Myog, Tnnt1, Ckm, and Mylpf are bound equally well by both Mef2D $\alpha 1$ and Mef2D $\alpha 2$ in differentiating myotubes (Supplemental Fig. 8). RNA-seq analysis of transcripts that are up-regulated at $24 \mathrm{~h}$ of differentiation when expression of Mef2D isoforms is induced in C2 cells identified 227 genes activated by Mef2D $\alpha 2$ (Supplemental Table 1). Combining the RNAseq data set with Mef2D peaks within $50 \mathrm{~kb}$ of the transcription start site identified 107 genes whose expression may be directly modulated by Mef2D $\alpha 2$ (Supplemental Table 1; Supplemental Fig. 10). Consistent with Mef2D $\alpha 2$ modulating the late muscle gene expression program, GO analysis of putative Mef2D $\alpha 2$ direct target genes showed a highly significant enrichment for genes involved in muscle development and muscle contraction (Supplemental Fig. 10A). Importantly, although Mef2D $\alpha 1$ bound to most of the direct transcriptional targets of Mef2D $\alpha 2$, exogenous expression of the ubiquitous Mef2D isoform did not lead to a significant increase in expression at any of these genes (Supplemental Table 1; Supplemental Fig. 10B). Similarly, ChIP-qPCR analysis of Mef2D isoform binding in proliferating myoblasts demonstrates that both Mef2D $\alpha 1$ and Mef2D $\alpha 2$ bind to the Myh3 and Mylpf promoters (Supplemental Fig. 6B), although only Mef2D $\alpha 2$ binding gives rise to transcriptional activation. Taken together, these results demonstrate that altered DNA binding is not the major determinant of the differential activation of transcription by Mef2D isoforms.

\section{The muscle-specific Mef2D $\alpha 2$ isoform preferentially interacts with coactivator complexes}

To assess the possibility that Mef2D $\alpha 1$ and Mef2D $\alpha 2$ associate with alternate coregulatory proteins to regulate muscle gene expression, we performed a proteomic analysis of proteins that interact with each Mef2D isoform. Initially, we examined proteins that interact with recombinant Mef2D $\alpha 1$ and Mef2D 2 2. In a pull-down assay, Flag-tagged Mef2D $\alpha 1$ or Mef2D $\alpha 2$ proteins were used as bait to isolate interacting proteins from nuclear extracts prepared from myotubes (Supplemental Fig. 11). Mass spectrometry analysis of Mef2D $\alpha 1$ - or Mef2D $\alpha 2$ interacting proteins (Table 1) identified components of the coactivator Ash2L methyltransferase complexes, corepressor HDAC deacetylase complexes, and the SWI/SNF ATP-dependent chromatin remodeling complexes that have previously been reported to associate with Mef2D (Ohkawa et al. 2006, 2007; Rampalli et al. 2007; Du et al. 2008). Qualitative analysis of the identified peptides showed that recombinant Mef2D $\alpha 1$ and Mef2D $\alpha 2$ interact with the same sets of proteins (Table 1). This finding suggests that the exchange of mutually exclusive exons in Mef2D does not generate proteins with distinct protein-protein interaction domains. However, the experiment was performed with recombinant Mef2D isoforms. To determine whether Mef2D $\alpha 1$ and Mef2D $\alpha 2$ isoforms also interact with the same sets of proteins in their native cellular context, we immunoprecipitated endogenous Mef2D $\alpha 1$ and Mef2D $\alpha 2$ proteins from myotube extracts and tested the interaction with the previously identified cofactors by Western blot (Fig. 4A). Strikingly, in these conditions, we observed differential interactions for Mef2D $\alpha 1$ versus Mef2D $\alpha 2$. Precisely, the muscle-specific Mef2D $\alpha 2$ interacts with the Ash2L coactivator complex but not the repressive HDAC4 and HDAC9 proteins. Conversely, the ubiquitously expressed Mef2D $\alpha 1$ does not associate with 
A

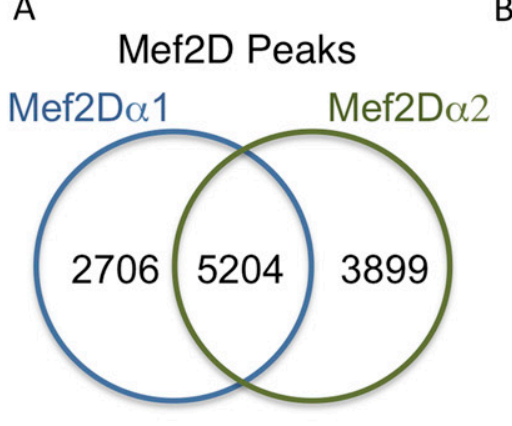

B

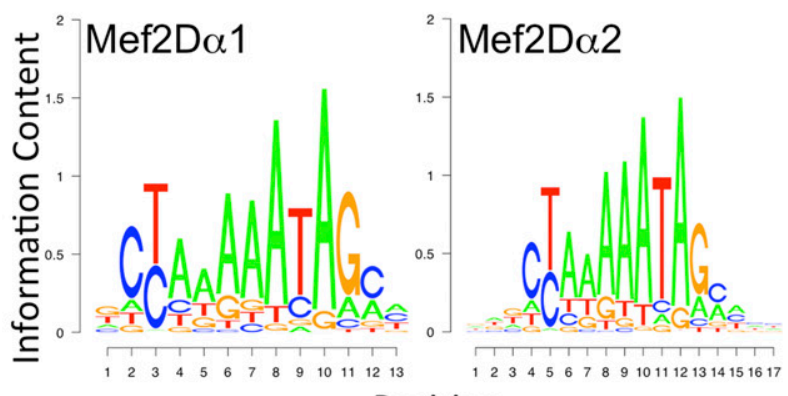

C

\begin{tabular}{|l|c|c|}
\hline Gene Ontology Analysis & \# of Genes & $p$-value \\
\hline Biological Process & $40 / 198$ & $1.06 \mathrm{E}-09$ \\
\hline muscle tissue development & $36 / 176$ & $4.43 \mathrm{E}-09$ \\
muscle organ development & $77 / 559$ & $9.49 \mathrm{E}-09$ \\
circulatory system development & $25 / 105$ & $1.11 \mathrm{E}-08$ \\
muscle cell development & $13 / 33$ & $1.15 \mathrm{E}-07$ \\
cellular component morphogenesis & $75 / 606$ & $1.14 \mathrm{E}-06$ \\
regulation of phosphorus metabolic process & $104 / 939$ & $2.20 \mathrm{E}-06$ \\
positive regulation of nitrogen compound metabolic process & $83 / 728$ & $3.18 \mathrm{E}-06$ \\
phosphorus metabolic process & $72 / 598$ & $4.67 \mathrm{E}-06$ \\
\hline
\end{tabular}

D

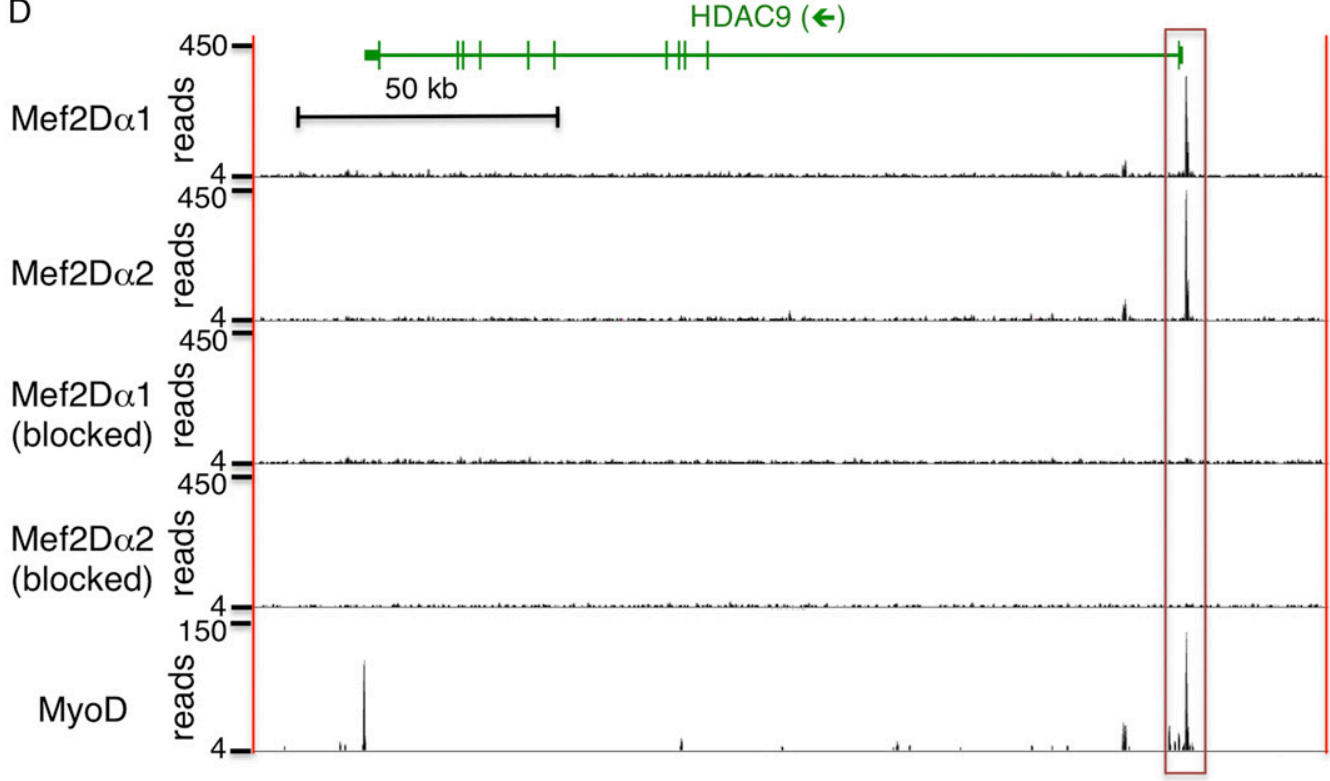

Figure 3. Mef2D isoforms bind a largely overlapping set of genes involved in muscle development. ChIP-seq analysis was performed on $\mathrm{C} 2$ myotubes that had been differentiated for $120 \mathrm{~h}$. Chromatin was immunoprecipitated with antibodies recognizing either Mef2D $\alpha 1$ or Mef2D $\alpha 2$. To control for nonspecific binding to the antibodies, control ChIP-seq experiments were performed with antibodies that were preincubated with peptides corresponding to the epitope and labeled as Mef2D $\alpha 1$ (blocked) or Mef2D $\alpha 2$ (blocked). (A) Venn diagram showing the overlap between the 9103 Mef2D 22 peaks and the 7910 Mef2D $\alpha 1$ peaks identified by ChIP-seq. (B) Logo diagrams of the position weight matrix of the sequence generated based on the enrichment of Mef2-binding sites observed in peaks bound by either Mef2D $\alpha 1$ or Mef2D $\alpha 2$. $(C)$ The top GO terms for biological processes (BP) are shown for the group of 5204 genes that are bound equally well by both Mef2D $\alpha 1$ and Mef2D $\alpha 2$. (D) The sequencing reads are mapped across the HDAC9 locus for ChIP experiments performed using Mef2D $\alpha 1$, Mef2D $\alpha 2$, Mef2D $\alpha 1$ (blocked), Mef2D $\alpha 2$ (blocked), and MyoD. Peaks for both Mef2D $\alpha 1$ and Mef2D $\alpha 2$ that overlap with MyoD binding are observed in the proximal promoter region (boxed in red).

Ash2L but interacts strongly with HDAC4 and HDAC9. Therefore, in contrast to their recombinant counterparts, endogenous Mef2D isoforms interact with distinct protein complexes in differentiating myotubes-Mef2D $\alpha 1$ interacts with corepressors, while Mef2D $\alpha 2$ interacts with coactivators. 
Table 1. Proteomics analysis of Mef2D $\alpha 1$ - and Mef2D $\alpha 2$ interacting proteins

\begin{tabular}{|c|c|c|c|c|}
\hline \multirow[b]{2}{*}{ Identified proteins } & \multicolumn{2}{|c|}{ Mef $2 \mathrm{D} \alpha 1$} & \multicolumn{2}{|c|}{ Mef2D $\alpha 2$} \\
\hline & Peptides & $\begin{array}{c}\text { Mascot } \\
\text { score }\end{array}$ & Peptides & $\begin{array}{c}\text { Mascot } \\
\text { score }\end{array}$ \\
\hline \multicolumn{5}{|l|}{ Transcription factors } \\
\hline Mef2D & 35 & 2848 & 24 & 1535 \\
\hline Myogenin & 4 & 119 & 3 & 74 \\
\hline Jun & 4 & 217 & 5 & 208 \\
\hline Jun-B & 4 & 197 & 4 & 275 \\
\hline Maf-G & 4 & 78 & 2 & 49 \\
\hline $\mathrm{Pbx} 3$ & 2 & 39 & - & - \\
\hline \multicolumn{5}{|l|}{ Ash2L complex } \\
\hline Ash2L & 6 & 89 & 10 & 145 \\
\hline WDR5 & 4 & 185 & 8 & 180 \\
\hline DPY-30 & 2 & 85 & - & - \\
\hline $\mathrm{HCFC1}$ & 4 & 88 & 2 & 54 \\
\hline \multicolumn{5}{|l|}{ HDAC complexes } \\
\hline HDAC2 & - & - & 3 & 23 \\
\hline SAP18 & 1 & 25 & 1 & 21 \\
\hline SAP30 & 1 & 26 & 1 & 31 \\
\hline $\mathrm{RbBP} 4$ & 9 & 261 & 11 & 341 \\
\hline $\mathrm{RbBP7}$ & 7 & 198 & 8 & 249 \\
\hline \multicolumn{5}{|l|}{ SWI/SNF complex } \\
\hline BRG1 & - & - & 2 & 38 \\
\hline BAF250a/Aridla & 15 & 236 & 9 & 50 \\
\hline BAF180 & 1 & 44 & - & - \\
\hline BAF170 & 18 & 455 & 15 & 345 \\
\hline BAF155 & 18 & 400 & 12 & 202 \\
\hline BAF60c & 10 & 359 & 14 & 409 \\
\hline BAF57 & 7 & 296 & 5 & 223 \\
\hline Brd7 & 2 & 53 & 2 & 37 \\
\hline
\end{tabular}

Recombinant Flag-tagged Mef2D $\alpha 1$ and Mef2D $\alpha 2$ proteins were used as bait to pull down interacting proteins from nuclear extracts prepared from C2 myotubes. Proteins interacting with the Mef2D isoforms were separated by SDS-PAGE and analyzed by mass spectrometry as described in the Supplemental Material. (Peptides) The number of unique peptides identified for each protein; (mascot score) the confidence in the proper identification of the protein; $(-)$ no peptides were identified for that particular protein.

\section{Mef2D $\alpha 2$ protein evades inhibitory protein kinase $A$ (PKA) signaling}

Based on the difference in protein-binding specificity observed between recombinant and endogenous proteins, we reasoned that the altered interactions could be modulated by post-translational modifications (PTMs). Indeed, several PTMs have been shown to regulate Mef2D function in vivo (Potthoff and Olson 2007). Important to transcriptional activation, phosphorylation of Mef2D by p38 $\alpha$ MAPK (p38 $)$ at positions Thr308 (T308) and T315 (see Fig. 4B) is required for recruitment of the Ash2L coactivator complexes to muscle genes (Rampalli et al. 2007). We therefore examined the propensity of Mef2D $\alpha 1$ and Mef2D $\alpha 2$ to be phosphorylated by p38 $\alpha$. Using recombinant proteins, in vitro kinase assays demonstrate that both Mef2D $\alpha 1$ and Mef2D $\alpha 2$ are efficiently phosphorylated by p38 $\alpha$ (Fig. 4C). Having observed that the activating phosphorylation of Mef2D by p $38 \alpha$ occurs in both Mef2D $\alpha 1$ and Mef2D $\alpha 2$, we next explored the possibility that the isoforms may be differentially affected by inhibitory PTMs. Transactivation by Mef2D is known to be inhibited through phosphorylation of Ser119 (S119) and S190 by PKA, which enables an association with class II HDACs (Du et al. 2008). Importantly, alternative exon usage in Mef2D $\alpha 2$ removes the S119 residue targeted by PKA in the Mef2D $\alpha 1$ isoform (see Fig. 4B). Thus, we examined whether the Mef2D $\alpha 1$ and Mef2D $\alpha 2$ isoforms are differentially phosphorylated by PKA. Consistent with previous observations (Du et al. 2008), Mef2D $\alpha 1$ is strongly phosphorylated by PKA (Fig. 4C). In contrast, Mef2D $\alpha 2$ is resistant to phosphorylation by PKA even though the phosphorylation target site S189(190) remains intact (Fig. 4C). These findings confirm the hypothesis that Mef2D isoforms are differentially modified by PTMs. Thus, overall, our results show that alternative exon usage regulates the ability of Mef2D protein to be phosphorylated by PKA.

\section{Mutation of PKA phosphorylation sites allows Mef2D 1 to activate myogenic transcription}

To better understand the importance of the differential phosphorylation of Mef2D isoforms by PKA, we examined how PKA activity changes during differentiation. Previous studies have shown that PKA signaling is required at various stages of myogenesis to mediate CREBdependent gene expression (Berdeaux and Stewart 2012). Using antibodies that recognize CREB phosphorylated at S133 to measure PKA activity, we observed that the PKA phosphorylates CREB in proliferating myoblasts, and the kinase remains active as myoblasts transition to myotubes (Fig. 4D). Thus, consistent with the requirement for activated CREB in myogenesis (Berdeaux and Stewart 2012), PKA activity is maintained throughout myotube formation.

The fact that PKA activity is maintained throughout differentiation suggests that the differential propensity to be phosphorylated by PKA could be responsible for the differential interaction of Mef2D isoforms with corepressor versus coactivators. To test this possibility, we generated the mutant Mef2D $\alpha 1(\mathrm{~S} 119 \mathrm{~A} / \mathrm{S} 190 \mathrm{~A})$, which is resistant to phosphorylated by PKA (Du et al. 2008). Similar to results observed with the endogenous protein, overexpressed Mef2D $\alpha 1$ did not interact with Ash2L but showed a strong interaction with HDAC9 (Fig. 5A). However, inhibition of phosphorylation by PKA using Mef2D $\alpha 1$ (S119/S190A) led to a marked reduction in the interaction between the mutant protein and the repressive HDAC9 protein. Surprisingly, the loss in interaction with HDAC9 is concurrent with the appearance of an association with the coactivator Ash2L that normally interacts with Mef2D $\alpha 2$. Thus, phosphorylation of Mef2D proteins by PKA is a primary determinant of the interaction with transcriptional coactivators or corepressors.

Finally, we examined whether the switch in Mef2D isoforms could represent a requirement for the transcription factor to evade inhibitory PKA signaling to mediate 


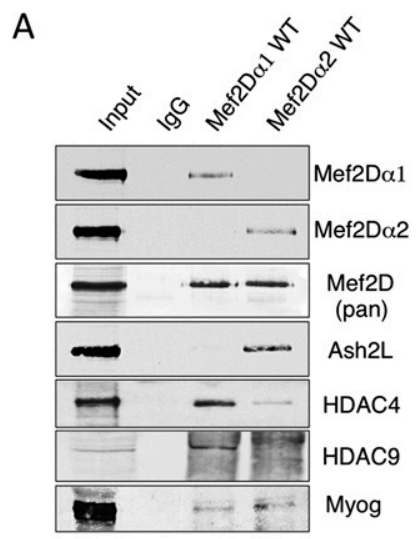

B

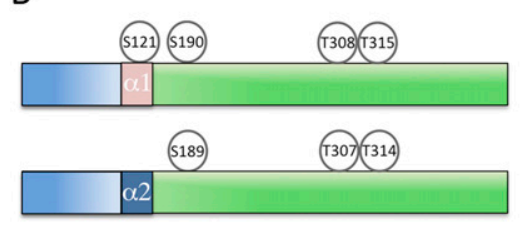

C

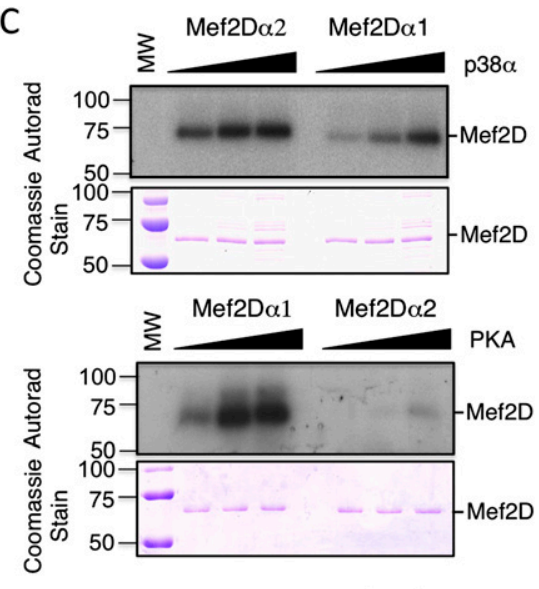

D

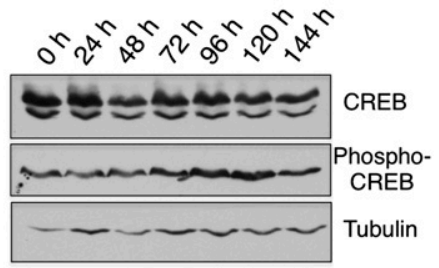

Figure 4. The muscle-specific isoform of Mef $2 \mathrm{D} \alpha 1$ isoform is resistant to phosphorylation by PKA. (A) The Ash2L complex preferentially interacts with the Mef2D $\alpha 2$ isoform. Nuclear extracts were prepared from C2 cells that had been induced to differentiate for 120 h. Endogenous Mef2D proteins were immunoprecipitated with antibodies recognizing Mef2D $\alpha 1$ or Mef2D $\alpha 2$ or with nonspecific IgG. Western blots were performed using the antibodies indicated. $(B)$ Schematic representation of the positioning of serine residues phosphorylated by PKA and threonine residues phosphorylated by p38 $\alpha$ MAPK in Mef2D $\alpha 1$ (and the equivalent residues in Mef2D $\alpha 2$ ). (C) In vitro kinase assays were performed using either purified p38 $\alpha$ MAPK (top panels) or PKA (bottom panels) in the presence of either Mef2D $\alpha 1$ or Mef2D $\alpha 2$. Mef2D proteins (100 ng) were incubated with varying concentrations of recombinant $\mathrm{p} 38 \alpha$ MAPK or PKA for 30 min in the presence of $4 \mu \mathrm{Ci}$ of $\gamma\left[{ }^{32} \mathrm{P}\right]$-ATP. Proteins were then separated by SDS-PAGE and exposed to autoradiographic (Autorad) film. Parallel reactions lacking $\gamma\left[{ }^{32} \mathrm{P}\right]$-ATP were separated by SDS-PAGE and stained with Coomassie blue. $(D)$ PKA activity in C2 cells was determined at different stages of differentiation by examining the amount of phosphorylated CREB present in whole-cell extracts. Western blots were performed on whole-cell extracts using the antibodies indicated.

activation of late gene transcription. Using RT-qPCR, we observed that, unlike wild-type Mef2D $\alpha 1$, the PKA phosphorylation mutant Mef2D 1 1(S119/S190A) gives rise to a strong activation of both Myh3 and Mylpf expression (Fig. 5B). Consistent with the differential ability to interact with coregulatory proteins, ChIP analysis showed that expression of Mef2D $\alpha 2$ or Mef2D $\alpha 1$ (S119/S190A) leads to an enrichment of Ash2L at both the Mylpf and Myh3 gene promoters, while expression of Mef2D 1 results in an increased enrichment of HDAC9 at these same loci (Fig. 5C). These findings demonstrate that Mef2D $\alpha 1$ (S119/S190A) and Mef2D $\alpha 2$ both activate expression of late muscle genes through the recruitment of coactivator proteins led us to examine whether the inability of the ubiquitously expressed Mef2D $\alpha 1$ protein to stimulate myotube formation may be due to PKA phosphorylation. As observed above, immunofluorescence shows that myoblasts expressing Mef2D $\alpha 1$ are resistant to the formation of $\mathrm{MHC}^{+}$myotubes at $48 \mathrm{~h}$ of differentiation (Fig. 5D). In contrast, expression of the PKA mutant Mef2D $\alpha 1$ (S119/S190) gave rise to $\mathrm{MHC}^{+}$ myotubes with a level of differentiation similar to that observed with Mef2D $\alpha 2$ (Fig. 5D; Supplemental Fig. 12). This demonstrates that the differential ability of Mef2D $\alpha 1$ and Mef2D 22 to drive myogenesis depends on their propensity for PKA-mediated phosphorylation. Thus, our results reveal a new role for alternative use of mutually exclusive exons in generating distinct transcription factors that have antagonizing roles in regulating differentiation based on their susceptibility to cell signaling-induced PTMs.

\section{Discussion}

Alternative splicing of transcription factors has been shown to direct tissue-specific gene expression by modifying the specificity of DNA-binding element recognition of the protein, resulting in the activation of an alternate set of genes (Reed et al. 2010; Gabut et al. 2011). Performing the first comparative genome-wide analysis of alternate transcription factor isoforms, we determined that the generation of a muscle-specific Mef2D $\alpha 2$ isoform does not alter the DNA-binding element recognition of the transcription factor compared with the more ubiquitous Mef2D $\alpha 1$ isoform. Instead, we found that alternate usage of mutually exclusive exons plays a crucial role in controlling the transcriptional activity of Mef2D during differentiation by altering its susceptibility to phosphorylation by PKA. Specifically, the Mef2D $\alpha 1$ isoform that is phosphorylated by PKA acts as a transcriptional repressor, while the PKA-resistant Mef2D $\alpha 2$ isoform activates late muscle gene expression. Thus, we identified a previously unappreciated mechanism by which a ubiquitously expressed transcription factor undergoes tissue-specific alternative exon usage to permit temporal activation of gene expression during differentiation.

A role for the Mef2 family of proteins in modulating gene expression in response to cell signaling pathways is well established (Potthoff and Olson 2007). Among the signaling pathways known to regulate Mef2 activity in development, PKA and p38 MAPK signaling are known to converge on Mef2D function during myogenesis. Studies using reagents that did not distinguish between 

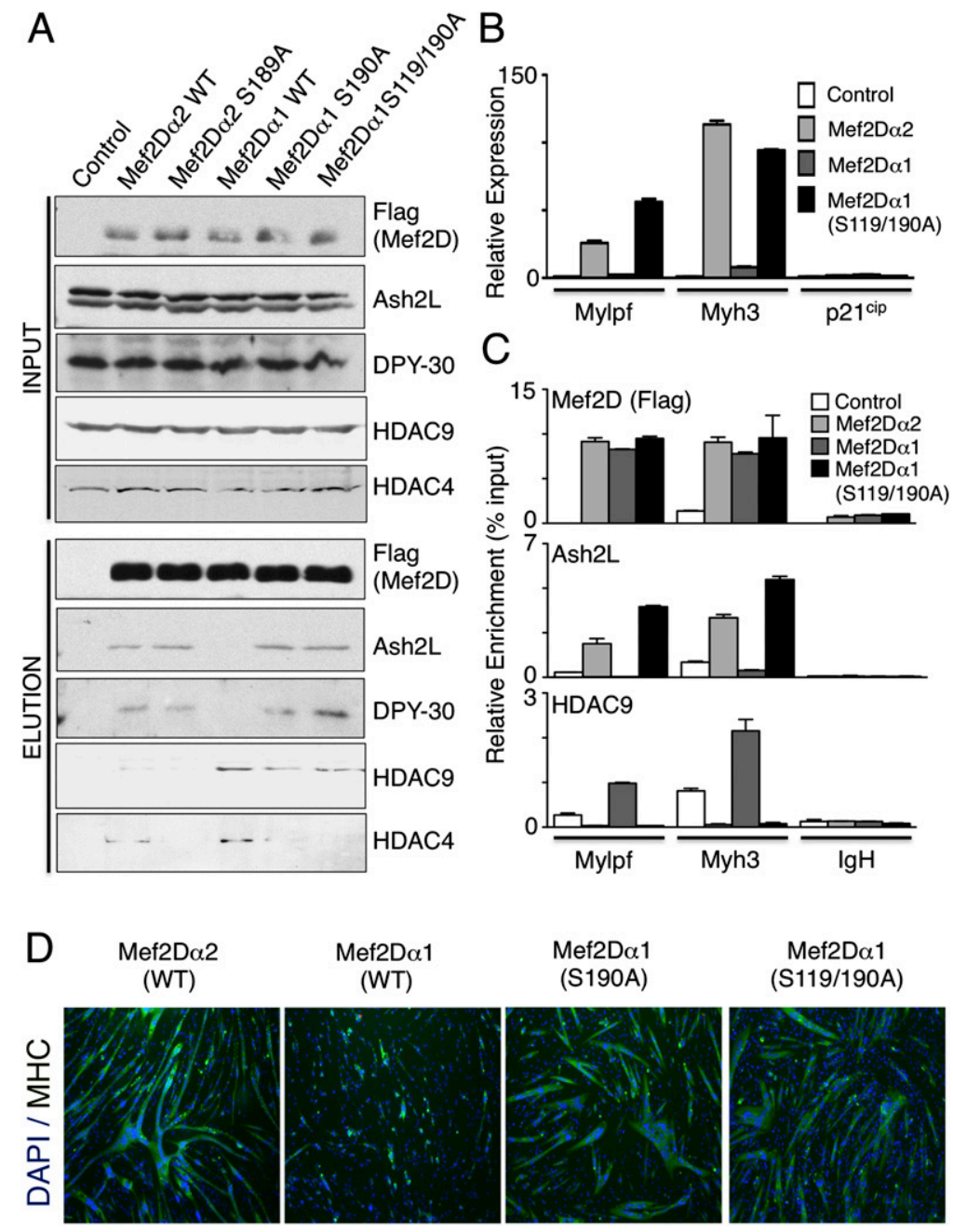

Figure 5. Mutation of PKA phosphorylation sites in Mef2D $\alpha 1$ facilitates its ability to activate MHC expression. (A) Ash2L complex interacts with forms of Mef2D that are not phosphorylated by PKA. Nuclear extracts were prepared from C2iFRT lines induced to express a Flag-tagged version of either Mef2D $\alpha 2(\mathrm{WT}), \quad M e f 2 \mathrm{D} \alpha 2(\mathrm{~S} 189 \mathrm{~A}), \operatorname{Mef} 2 \mathrm{D} \alpha 1(\mathrm{WT})$, Mef2D 1 (S190A), or Mef2Do1(S119/190A) that had been incubated in differentiation medium for $48 \mathrm{~h}$. Western blots were performed using the antibodies indicated. $(B, C)$ C2iFRT cells induced to express either Mef2D $\alpha 2(\mathrm{WT}), \operatorname{Mef} 2 \mathrm{D} \alpha 1(\mathrm{WT})$, Mef2D $\alpha 1(S 190 A)$, or Mef2D 1 1(S119/190A) were incubated in differentiation medium for $24 \mathrm{~h} .(B)$ Total RNA was converted to cDNA and analyzed using qPCR with primers specific for Myh3, Mylpf, p21 ${ }^{\mathrm{CIP}}$, or the ubiquitously expressed DDX5 mRNA. Values are expressed as the mean \pm SEM relative to the internal control DDX5 where the expression in the control was normalized to $1 . n=3$. $(C)$ Cross-linked chromatin from C2iFRT cells expressing exogenous Flag-tagged Mef2D proteins were subjected to ChIP analysis using antibodies directed against the Flag epitope. Immunopurified DNA was quantitated by qPCR using probes that recognize the promoter of the Myh3 or Mylpf genes or the upstream enhancer of IgH. (D) C2iFRT cells induced to express Mef2D $\alpha 2(\mathrm{WT}), \operatorname{Mef} 2 \mathrm{D} \alpha 1(\mathrm{WT}), \mathrm{Mef} 2 \mathrm{D} \alpha 1(\mathrm{~S} 190 \mathrm{~A})$, or Mef2D 1 (S119/190A) were incubated in differentiation medium for $48 \mathrm{~h}$. Immunofluorescence was performed using antibodies recognizing MHC (green) or DAPI (blue) as indicated.
Mef2D $\alpha 1$ and Mef2D $\alpha 2$ have shown that PKA represses Mef2D function (Du et al. 2008), while p38 $\alpha$ stimulated its transactivation (Penn et al. 2004; Rampalli et al. 2007). In the case of $\mathrm{p} 38 \alpha$, phosphorylation of Mef2D allows the recruitment of Ash2L/MLL2-containing methyltransferase complexes to establish trimethylation of histone $\mathrm{H} 3$ (H3K4me3) within promoters of MyoD target genes to facilitate transcription (Rampalli et al. 2007; Aziz et al. 2010). In contrast, PKA-mediated phosphorylation of Mef2D engages an interaction between the transcription factor and class II HDACs to mediate transcriptional repression (Du et al. 2008). The use of antibodies that allow us to distinguish between Mef2D $\alpha 1$ and Mef2D $\alpha 2$ confirm these original findings, as Mef2D proteins can interact with both corepressors and coactivators during myogenesis. However, our analysis of specific isoforms demonstrates that it is not the same Mef2D molecules that interact with these antagonizing coregulatory molecules. Instead, we found that the ubiquitous Mef2D $\alpha 1$ interacts with the corepressors HDAC4 and HDAC9 through an interaction enabled by PKA-mediated phosphorylation. Splicing of the Mef2D transcripts during myogenesis then generates a muscle-specific protein that is resistant to PKA phosphorylation and associates with the Ash2L coactivator complex. This represents a significant advance in our understanding of myogenesis, as previous studies showed that PKA phosphorylates Mef2D to block its ability to drive myogenesis (Du et al. 2008). This inhibitory effect of PKA on Mef2D function was perplexing due to the fact that PKA signaling is required in myogenesis to facilitate CREB-dependent transactivation and myoblast fusion (Chen et al. 2005; Mukai and Hashimoto 2008; Stewart et al. 2011; Berdeaux and Stewart 2012). Our finding that Mef2D undergoes a splicing event during differentiation that renders the transcription factor resistant to PKA inhibition resolves this paradox and highlights the need to study isoform-specific functions of proteins during development.

Resolving the paradox of continued PKA signaling during myogenesis is an important finding that identifies an alternate mechanism that evolved for cells to overcome inhibitory signaling pathways during differentiation. Indeed, common models for development describe the down-regulation of signaling pathways that are inhibitory to differentiation concomitant with the up-regulation of prodifferentiation signals to permit progression toward a 
new cell fate (Iglesias-Bartolome and Gutkind 2011). However, PKA signaling is both promyogenic through phosphorylation of CREB (Chen et al. 2005; Mukai and Hashimoto 2008; Stewart et al. 2011; Berdeaux and Stewart 2012) and inhibitory to myogenesis through phosphorylation of Mef2D (Du et al. 2008). Thus, the muscle-specific switch in exon usage for Mef2D $\alpha 2$ evolved to evade the inhibitory effects of PKA signaling, allowing transcriptional activation of Mef2D target genes in the presence of promyogenic PKA signaling. While we showed that the exon switching is used by Mef2D to evade phosphorylation by PKA, it remains to be determined how extensively splicing is used to evade signaling pathways in development. However, we propose that isoform switching may be a widely used mechanism to modify susceptibility of a protein to cell signaling, as recent studies suggest that tissue-specific exons often encode disordered peptides that are enriched for sites of phosphorylation (Buljan et al. 2012).

Profiling of Mef2D $\alpha 1$ and Mef2D $\alpha 2$ protein levels during myogenesis demonstrated a temporal control of isoform abundance where a clear switch in exon usage allows accumulation of the muscle-specific Mef2D $\alpha 2$ starting at an intermediate stage of differentiation. The timing of this splicing event suggests a major role for Mef2D $\alpha 2$ in ensuring the proper temporal activation of late gene expression during myogenesis. Previous studies of myogenesis have identified a role for Mef2D in establishing the proper temporal activation of a subset of muscle genes (Penn et al. 2004). Furthermore, studies in zebrafish have demonstrated that Mef2 proteins are important to the activation of the late stages of the myogenic program (Hinits and Hughes 2007). Based on these observations, we propose a model for the temporal regulation of late muscle gene expression through Mef2D isoform switching (Fig. 6). In this model, Mef2D $\alpha 1$ is the predominant isoform of the transcription factor expressed in the early myogenesis. Active PKA signaling in the cell catalyzes phosphorylation of Mef2D $\alpha 1$ to establish an HDAC-dependent transcriptional repression at late muscle genes. As differentiation progresses, Mef2D transcripts undergo alternative splicing, leading to the establishment of two distinct isoforms: the ubiquitous Mef2D $\alpha 1$ isoform that continues to act as a transcriptional repressor and a muscle-specific Mef2D $\alpha 2$ isoform that is resistant to PKA signaling and permits the transcriptional activation of the late muscle gene expression program. As our ChIP-seq studies have shown that Mef2D $\alpha 1$ and Mef2D 22 bind to overlapping subsets of target genes in the late stages of myogenesis, we propose that an ongoing competition between the two isoforms
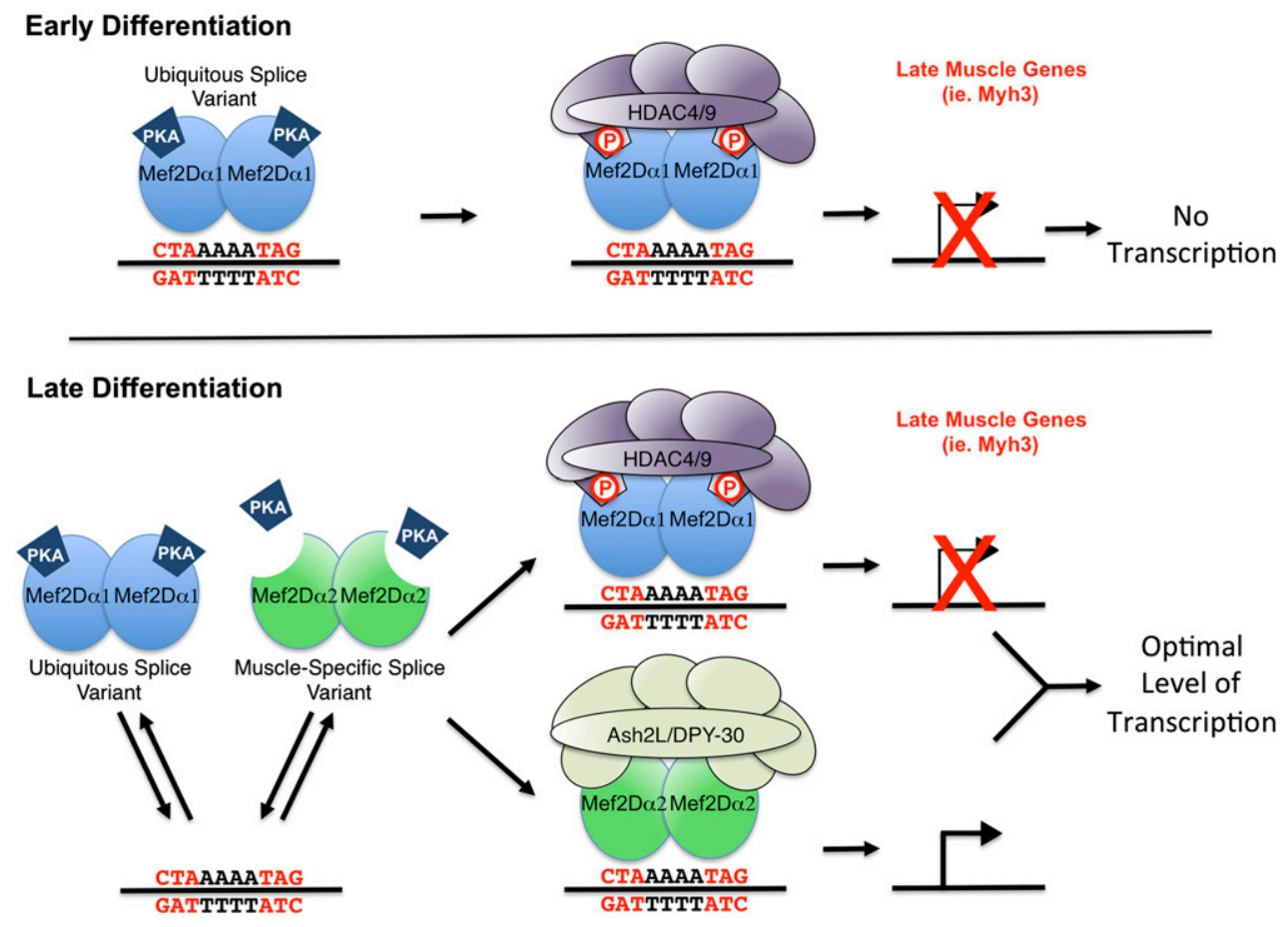

Figure 6. Model for the temporal regulation of late muscle gene expression through the use of alternate exons. During the early stages of differentiation, the ubiquitous isoform of Mef2D (Mef2D $\alpha 1)$ is expressed almost exclusively. The majority of the Mef2D 11 are phosphorylated by active PKA signaling, leading to a recruitment of class II HDAC4/9 onto muscle promoters to maintain a transcriptionally repressed state. At later stages of differentiation, alternative splicing of Mef2D transcripts gives rise to a second population of Mef2D proteins (Mef2D $\alpha 2)$. The Mef2D $\alpha 2$ isoform, which cannot be phosphorylated by PKA, competes with Mef2D $\alpha 1$ for binding to target genes. While binding of PKA-phosphorylated Mef2D $\alpha 1$ leads to recruitment of class II HDACs to repress the promoter, Mef2D $\alpha 2$ (and unphosphorylated Mef2D $\alpha 1$ ) leads to recruitment of the Ash2L complexes to muscle promoters to activate gene expression. This competition would result in an ongoing dynamic exchange of repressive and activating Mef2D transcription factors that is proposed to act as a rheostat to ensure an optimal level of transcription to mediate formation of function myotubes. 
exists for binding to Mef2 elements within the promoter, leading to the recruitment of both coactivator and corepressor proteins to muscle genes. Such a competition has previously been demonstrated for glucocorticoid receptors, where continuous cycling on and off the MMTV promoter (McNally et al. 2000) allows for dynamic exchange of transcription factor isoform binding at a single genomic site (Voss et al. 2011). Similarly, a dynamic exchange of Mef2D $\alpha 1$ and Mef2D $\alpha 2$ binding in differentiating myotubes would permit the alternate association of coactivators and corepressors at the promoter region of late muscle genes to achieve optimal expression levels required for healthy muscle development and function. This model is completely consistent with previous genomewide studies that demonstrated that HDAC and histone acetyltransferase proteins are both highly enriched at actively transcribed genes to ensure efficient gene expression (Wang et al. 2009).

In conclusion, we showed that the muscle-specific isoform of Mef2D plays a key role in establishing the late muscle gene expression program. This role is enabled through a temporally regulated switch in exon usage that allows Mef2D to evade inhibitory phosphorylation by PKA. Thus, we identified a novel role for the alternate use of mutually exclusive exons to modulate the transition in function of a transcription factor from repressor to activator through altered susceptibility to an inhibitory PTM.

\section{Materials and methods}

\section{Antibodies}

Rabbit polyclonal antibodies that specifically recognize the alternate isoforms of Mef2D were generated by Genscript using keyhole limpet hemocyanin-coupled peptides-CELDGLFRRYGST (for Mef2D $\alpha$ 1) or CPQTEEKYKKIDEEF (for Mef2D $\alpha 2$ ). The pan-Mef2D mouse monoclonal antibody was purchased from BD Biosciences (catalog no. 610774). Ash2L antibody has been previously described (Demers et al. 2007). The DPY-30 antibody was generated in a rabbit using a His-tagged full-length mouse DPY-30 protein as antigen. The following antibodies recognizing different forms of MHC were obtained from the Developmental Studies Hybridoma Bank: embryonic MHC (MF20), MHC-I (A4.840), MHC-IIa (SC-71), and MHC-IIb (BF-F3). CREB (Santa Cruz Biotechnology, catalog no. SC-186), phospho-CREB (Ser133) (Cell Signaling, catalog no. 9191), HDAC4 (Cell Signaling, catalog no. 2072), HDAC9 (Abcam, catalog no. 109446), tubulin (Developmental Studies Hybridoma Bank, catalog no. 6G7), and myogenin (Santa Cruz Biotechnology, catalog no. sc12732) antibodies are commercially available.

\section{Cell culture}

C2 myoblasts (Yaffe and Saxel 1977) were maintained in Dulbecco's modified medium (4.5 g/L glucose) supplemented with $10 \%$ fetal calf serum, $4 \mathrm{mM}$ L-glutamine, and $1.5 \mathrm{~g} / \mathrm{L}$ sodium bicarbonate while not allowing cell density to achieve $>80 \%$ confluent. To differentiate myoblasts, cells were allowed to achieve $90 \%$ confluency prior to a shift in culture medium to Dulbecco's modified medium containing $2 \%$ horse serum with insulin $(10 \mu \mathrm{g} / \mathrm{mL})$ and transferrin $(10 \mu \mathrm{g} / \mathrm{mL})$. Differentiation medium was changed every $24 \mathrm{~h}$ for up to $5 \mathrm{~d}$.
Generation of isogenic C2 lines expressing Mef2D isoforms

A C2 line containing a single insertion of an FRT recombination element was generated using the Flp-In system (Invitrogen). The C2iFRT cell line was then generated to constitutively express the Tet repressor by selecting for cells transfected with the pPyCAGIPTetR plasmid. Clones were then screened by immunofluorescence to ensure that they retained the potential to differentiate (express Myog and Myh3 while forming multinucleated myotubes) upon serum withdrawal. The cDNAs encoding Mef2D $\alpha 1$, Mef2D $\alpha 2$, Mef2D 2 (S189A), Mef2D 1 1(S190A), or Mef2D $\alpha 1$ (S119/190A) were cloned into the multiple cloning site of a modified pCDNA5/ FRT plasmid, where the CMV promoter was replaced by a CMV containing a Tet operator. Plasmids were transfected separately into C2iFRT cells and selected for hygromycin expression as outlined in the Flp-In system protocol. Batch cultures were then screened for their ability to differentiate (as described above) and Mef2D expression. Batch cell lines were termed C2iFRT-Mef2D $\alpha 1$, C2iFRTMef2D $\alpha 2$, C2iFRT-Mef2D $\alpha$ (S189A), C2iFRT-Mef2D $\alpha$ 1(S190A), or C2iFRT-Mef2D 1 1(S119/190A) based on the form of Mef2D that was exogenously expressed when induced with Dox at a concentration of $0.5 \mu \mathrm{M}$.

\section{ChIPs}

C2 myoblasts $\left(7 \times 10^{7}\right.$ cells $)$ were differentiated for $5 \mathrm{~d}$. Proteins were cross-linked to DNA for $30 \mathrm{~min}$ using $1 \%$ formaldehyde in Dulbecco's modified Eagle medium (DMEM) at $22^{\circ} \mathrm{C}$. After 30 min, the formaldehyde-containing medium was removed, and cells were washed with $125 \mathrm{mM}$ glycine in PBS for $10 \mathrm{~min}$ at $22^{\circ} \mathrm{C}$. Recovered cells were resuspended in a hypotonic lysis buffer $(25$ $\mathrm{mM}$ Hepes at $\mathrm{pH} 7.9,1.5 \mathrm{mM} \mathrm{MgCl} 2,10 \mathrm{mM} \mathrm{KCl}, 0.1 \% \mathrm{NP}-40$, $0.5 \mathrm{mM}$ DTT, $0.3 \mathrm{mM}$ PMSF). After $45 \mathrm{~min}$ on ice, myotubes were lysed using a 2-mL dounce homogenizer (B-pestle). Pellets recovered from homogenate were resuspended in nuclease buffer (50 mM Tris $\mathrm{HCl}$ at $\mathrm{pH} 7.5,140 \mathrm{mM} \mathrm{NaCl}, 5 \mathrm{mM} \mathrm{MgCl}_{2}, 1 \mathrm{mM}$ $\mathrm{CaCl}_{2}$ ). MNase (Sigma) was added to the chromatin at a concentration of $0.12 \mathrm{U}$ per $100 \mu \mathrm{g}$ of ribonucleic acids (measured as in Brand et al. 2008) and incubated for $5 \mathrm{~min}$ at $37^{\circ} \mathrm{C}$. Fragmentation of the DNA was stopped by the addition of one-tenth volume of MNase stop buffer (11 mM EDTA, 11 mM EGTA). The chromatin was then pelleted at $4000 \mathrm{rpm}$ for $10 \mathrm{~min}$ at $4^{\circ} \mathrm{C}$. The pellet was resuspended in shearing buffer $(50 \mathrm{mM}$ Tris $\mathrm{HCl}$ at $\mathrm{pH} 7.5,140 \mathrm{mM}$ $\mathrm{NaCl}, 1 \mathrm{mM}$ EDTA, $0.5 \%$ SDS, $1 \%$ Triton X-100) and sonicated using a Bioruptor for 80 cycles of $30 \mathrm{sec}$ on and $60 \mathrm{sec}$ off to further fragment the chromatin to obtain fragments of 150-300 base pairs (bp). After sonication, the chromatin was then centrifuged to pellet debris, and the supernatant was transferred to fresh Eppendorf tubes for immunoprecipitation with antibodies of interest as previously described (Seenundun et al. 2010). Libraries were generated from immunoprecipitated DNA as outlined in the Illumina DNA Library Construction kit protocol, except that 21 rounds of PCR were performed for amplification, and DNA fragments ranging from 200 to $350 \mathrm{bp}$ were isolated during size selection on agarose gel. DNA libraries were sequenced on an Illumina Hi-Seq 2000 at the McGill University and Génome Québec Innovation Center. The DNA sequences of primer sets used for ChIP-qPCR analysis are available on request.

\section{High-throughput DNA sequencing analysis}

Sequences were extracted using GAPipeline. Reads were aligned by Burrows-Wheeler alignment (BWA) to the mouse genome (mm9). Duplicate sequences were discarded to minimize the effects of PCR amplification. Each read was extended in the sequencing orientation to a total of 200 bases to infer the coverage at 
each genomic position. Peak calling was performed by an inhouse-developed $\mathrm{R}$ package as previously described (Palii et al. 2011; Fong et al. 2012). Motif analyses were performed using inhouse-developed Bioconductor package motifRG (Palii et al. 2011; Fong et al. 2012). To detect differential peaks between two samples, we first took the square root of the peak heights in both samples and fit a local regression curve on the difference of the two versus the mean. The residual is defined as the difference subtracted by the fitted value, which adjusts for mean-dependent bias. We fit another local regression model on squared residual versus mean to estimate mean-dependent variance. The $Z$-value is defined by the residual divided by standard deviation (square root of the estimated variance). This approach draws some similarity to the mean-dependent dispersion estimate by DESEQ (Anders and Huber 2010), except that we used a nonparametric approach instead of modeling by a negative binomial distribution. ChIP-seq data were deposited in Gene Expression Omnibus (GEO; accession no. GSE43223).

\section{RNA-seq}

C2iFRT-Mef2D $\alpha 1$, C2iFRT-Mef2D $\alpha 2$, or control (C2iFRT) cells were grown in proliferative conditions for $24 \mathrm{~h}$ in the presence of Dox. Near confluence, cells were then differentiated for a further $24 \mathrm{~h}$ in the presence of Dox. Total RNA was isolated from cells using the RNeasy minikit (Qiagen) using standard conditions. Library preparation and 100-bp paired-end RNA-seq were performed using standard Illumina procedures for the HiSeq 2000 platform.

The RNA-seq data were analyzed using the Bowtie, TopHat, CuffLinks, and CummeRbund software suite (Trapnell et al. 2012). Briefly, paired-end 100-bp reads were mapped to the $\mathrm{mm} 9$ genome (University of California at Santa Cruz) and known $\mathrm{mm} 9$ transcripts (RefSeq $\mathrm{mm} 9$ build37.2) using Bowtie version 2.0.2 (Langmead and Salzberg 2012) with default parameters. Splice junctions were determined from the mapped reads using TopHat version 2.0.6 (Trapnell et al. 2009) with default values with the exception of $-r=0$. Transcript assembly and quantification was performed with CuffLinks version 2.0.2 (Trapnell et al. 2010) using default values. Identification of differential gene expression was performed with the CummeRbund bioconductor package (http://compbio.mit.edu/cummeRbund). Genes with a $Q$-value $\leq 0.05$ were considered to be significant. RNA-seq data were deposited in GEO (accession no. GSE46363).

\section{Regeneration in mice}

Mouse studies were performed in accordance with the University of Ottawa regulations for animal care and handling. The left and right TA muscles of wild-type CD-1 mice (obtained from Charles River) were injured by injection of cardiotoxin as previously described (Liu et al. 2012). After $48 \mathrm{~h}$ of recovery, retrovirus expressing either Mef2D $\alpha 1$ or Mef2D $\alpha 2$ (multiplicity of infection [MOI] $1 \times 10^{6}$ ) was injected into one of the regenerating TA muscles, while the contralateral regenerating muscle was injected with a control retrovirus. After 3, 7, or $15 \mathrm{~d}$ of recovery, TA muscles were isolated and sectioned (12 $\mu \mathrm{m}$ thickness). For TA samples taken at $15 \mathrm{~d}$ of regeneration, the muscles were prepared for paraffin inclusion as described in Liu et al. (2012). For TA samples prepared at 3 and $7 \mathrm{~d}$ of regeneration, unfixed muscles were embedded in OCT compound, frozen in isopentane cooled with liquid nitrogen, and then sectioned ( $12 \mu \mathrm{m}$ thickness). To assess regeneration, cross-sections were prepared for histological examination on a ScanScope CS microscope (Aperio) after staining with hematoxylin and eosin. Changes in fiber size distribution between muscles injected with Mef2D-expressing virus and the control were determined using a nonparametric (Wilcoxon) test. Fiber type switching was assessed by immunofluorescence using antibodies recognizing different isoforms of MHC. After immunostaining, coverslips were mounted in mounting medium with DAPI (Vector Laboratories, Inc., H-1500) and imaged using a Zeiss LSM 510 Meta confocal microscope.

\section{Protein-protein interaction analysis}

Nuclear extracts were prepared from differentiated C2 myotubes (5 d) as previously described (Weil et al. 1979) with the following modifications. Nuclear pellets were resuspended in one pellet volume of modified buffer $\mathrm{C}(20 \mathrm{mM}$ Hepes at $\mathrm{pH} 7.6,1.5 \mathrm{mM}$ $\mathrm{MgCl}_{2}, 650 \mathrm{mM} \mathrm{KCl}$, benzonase [2.5 $\mathrm{U}$ per $10^{7}$ cells], $0.2 \mathrm{mM}$ PMSF, $0.5 \mathrm{mM}$ DTT, $5 \mathrm{mM} \beta$-glycerolphosphate, $1 \mathrm{mM}$ sodium orthovanadate) and rotated for $30 \mathrm{~min}$ at $4^{\circ} \mathrm{C}$. Nuclear homogenates were then diluted with one pellet volume of buffer $\mathrm{E}$ (20 mM Hepes at $\mathrm{pH} 7.6,1.5 \mathrm{mM} \mathrm{MgCl} 2,0.2 \mathrm{mM}$ EDTA, $1 \mathrm{mM}$ PMSF, $0.5 \mathrm{mM}$ DTT, $5 \mathrm{mM} \beta$-glycerolphosphate, $1 \mathrm{mM}$ sodium orthovanadate). Extracted proteins were recovered from the nuclei by centrifugation at $15,000 \mathrm{~g}$ for $30 \mathrm{~min}$ at $4^{\circ} \mathrm{C}$. Nuclear extracts were then quantitated for protein content prior to interaction studies.

For immunoprecipitation studies of endogenous proteins, $10 \mu \mathrm{g}$ of specific antibody or rabbit IgG were prebound to $20 \mu \mathrm{L}$ of protein A Dynabeads. Flag immunoprecipitations were performed using M2-agarose (Sigma). Mef2D-associated proteins were immunoprecipitated as previously described (Chaturvedi et al. 2012) and eluted by incubating with $5 \%$ acetic acid for $5 \mathrm{~min}$ at $22^{\circ} \mathrm{C}$. The acetic acid was removed from the recovered proteins by evaporation using a SpeedVac (Eppendorf) before proceeding with Western blot analysis.

In the pull-down studies, baculovirus-expressed Flag-Mef2D $\alpha 1$ or Flag-Mef2D $\alpha 2$ proteins were purified from Sf9 insect cells using M2-agarose (Sigma) as previously described (Rampalli et al. 2007). Briefly, M2-agarose-bound proteins were washed in stringent conditions $(500 \mathrm{mM} \mathrm{KCl})$ and then equilibrated in immunoprecipitation buffer $(20 \mathrm{mM}$ Hepes at $\mathrm{pH} 7.6,150 \mathrm{mM} \mathrm{KCl}, 1.5 \mathrm{mM}$ $\mathrm{MgCl}_{2}, 0.2 \mathrm{mM}$ EDTA, $0.2 \mathrm{mM}$ PMSF, $0.5 \mathrm{mM}$ DTT). FlagMef2D-bound M2 agarose beads were then incubated with nuclear extracts for $4 \mathrm{~h}$ at $4^{\circ} \mathrm{C}$. After washing, proteins were eluted with 3X-Flag peptide (Sigma) and separated by SDS-PAGE. After visualization with Coomassie blue, protein bands were excised for mass spectrometry analysis.

\section{Mass spectrometry}

Protein identification was performed at the Ottawa Hospital Research Institute Proteomics Core Facility (Ottawa, Canada). Gel bands were in-gel-digested according to the method of Shevchenko et al. (2006). Peptide extracts were concentrated by SpeedVac (Eppendorf) and resuspended in $0.1 \%$ trifluoroacetic acid. Peptides were loaded onto a peptide trap (Zorbax SB300-C18, Agilent) for $5 \mathrm{~min}$ at $15 \mu \mathrm{L} / \mathrm{min}$ using a Dionex UltiMate 3000 RSLC nano high-pressure liquid chromatography (HPLC). Peptides were eluted over a 60 -min gradient of $3 \%-45 \%$ acetonitrile with $0.1 \%$ formic acid at $0.3 \mu \mathrm{L} / \mathrm{min}$ onto a $10-\mathrm{cm}$ analytical column (New Objective Picofrit self-packed with Zorbax C18) and sprayed directly into a LTQ Orbitrap XL hybrid mass spectrometer using a nanospray source (Thermo Scientific). Mass spectra were acquired in a data-dependent fashion, with MS scans acquired in the Fourier Transform cell, while $\mathrm{MS}^{2}$ scans were acquired in the ion trap module.

Tandem mass spectrometry (MS/MS) spectra were matched against a custom database comprised of mouse sequences in SwissProt (2011_07 version of uniprot_sprot.fasta.gz from ftp:// 
ftp.uniprot.org) concatenated with a database of common contaminants or against the full SwissProt database using MASCOT 2.3.01 software (Matrix Science) with mass spectrometry tolerance of \pm 5 parts per million (ppm) and MS/MS tolerance of $0.6 \mathrm{Da}$. For standard Mascot search, oxidation of methionine, carbamidomethylation of cysteine, deamidation, protein N-terminal acetylation, and conversion of peptide N-terminal Glu or Gln to PyroGlu were allowed as potential protein modifications.

\section{Immunofluorescence}

Cells were fixed using $4 \%$ paraformaldehyde, permeabilized with $0.5 \%$ Triton X-100/PBS, and then blocked with $5 \%$ BSA in PBS. Primary antibodies were diluted with PBS $/ 5 \%$ BSA solution and incubated overnight at $4^{\circ} \mathrm{C}$. After washing, cells were incubated with secondary antibodies conjugated with Alexa Fluor 488 (Invitrogen, A11001) and/or Alexa Fluor 546 (Invitrogen, A11010). Prior to visualization, coverslips were mounted in mounting medium with DAPI (Vector Laboratories, Inc., H-1500). Images were taken using an Olympus FluoView FV1000 confocal microscope.

\section{Kinase assay}

Baculovirus-expressed Flag-Mef2D $\alpha 1$ or Flag-Mef2D $\alpha 2$ proteins were purified from Sf9 insect cells using M2-agarose (Sigma). Hisp38 $\alpha$ was purified from bacteria in its active (phosphorylated) form as previously described (Khokhlatchev et al. 1997). Active PKA was purchased from New England Biolabs. Recombinant Mef2D proteins (100 ng) were incubated with either His-p38 $\alpha$ $(80,250$, or $750 \mathrm{fmol})$ or PKA $(50,100$, or $200 \mathrm{U})$ in the presence of $1 \mathrm{pmol}(4 \mu \mathrm{Ci})$ of $\left[{ }^{32} \mathrm{P}\right] \gamma-\mathrm{ATP}$ in a buffer containing $10 \mathrm{mM}$ Hepes (pH 7.4), $50 \mathrm{mM} \mathrm{KCl}, 1.5 \mathrm{mM} \mathrm{MgCl} 2,10 \%$ glycerol, $0.5 \mathrm{mM}$ EGTA, and $10 \mathrm{mM} \beta$-glycerophosphate for $30 \mathrm{~min}$ at $37^{\circ} \mathrm{C}$. Reactions were then separated on $10 \%$ SDS-PAGE gels, dried, and exposed to autoradiographic film. Parallel reactions lacking $\left[{ }^{32} \mathrm{P}\right] \gamma-$ ATP were run on $10 \%$ SDS-PAGE gels and stained for protein content with Coomassie blue.

\section{Acknowledgments}

We thank Lynn Megeney and Alexandre Blais for critically reading the manuscript, Alexandre Montpetit for ChIP-seq and RNA-seq library sequencing, and Lawrence Puente for mass spectrometry analysis. This work was funded by the Canadian Institute of Health Research (MOP-77778 to F.J.D., and MOP89834 to M.B.) and National Institutes of Health (NIH National Institute of Arthritis and Musculoskeletal and Skin Diseases R01AR045113 to S.J.T.). S.S. and C.P. were funded by the Ontario Research Fund supporting the International Regulome Consortium. F.J.D. holds a Canada Research Chair in the Epigenetic Regulation of Transcription.

\section{References}

Anders S, Huber W. 2010. Differential expression analysis for sequence count data. Genome Biol 11: R106.

Aziz A, Liu QC, Dilworth FJ. 2010. Regulating a master regulator: Establishing tissue-specific gene expression in skeletal muscle. Epigenetics 5: 692-696.

Berdeaux R, Stewart R. 2012. cAMP signaling in skeletal muscle adaptation: Hypertrophy, metabolism, and regeneration. Am I Physiol Endocrinol Metab 303: E1-E17.

Bergstrom DA, Penn BH, Strand A, Perry RL, Rudnicki MA, Tapscott SJ. 2002. Promoter-specific regulation of MyoD binding and signal transduction cooperate to pattern gene expression. Mol Cell 9: 587-600.

Brand M, Rampalli S, Chaturvedi CP, Dilworth FJ. 2008. Analysis of epigenetic modifications of chromatin at specific gene loci by native chromatin immunoprecipitation (N-ChIP) of nucleosomes isolated using hydroxyapatite chromatography. Nat Protoc 3: 398-409.

Buljan M, Chalancon G, Eustermann S, Wagner GP, Fuxreiter M, Bateman A, Babu MM. 2012. Tissue-specific splicing of disordered segments that embed binding motifs rewires protein interaction networks. Mol Cell 46: 871-883.

Chaturvedi CP, Somasundaram B, Singh K, Carpenedo RL, Stanford WL, Dilworth FJ, Brand M. 2012. Maintenance of gene silencing by the coordinate action of the H3K9 methyltransferase G9a/KMT1C and the H3K4 demethylase Jarid1a/KDM5A. Proc Natl Acad Sci 109: 18845-18850.

Chen AE, Ginty DD, Fan CM. 2005. Protein kinase A signalling via CREB controls myogenesis induced by Wnt proteins. Nature 433: 317-322.

Demers C, Chaturvedi CP, Ranish JA, Juban G, Lai P, Morle F, Aebersold R, Dilworth FJ, Groudine M, Brand M. 2007. Activator-mediated recruitment of the MLL2 methyltransferase complex to the $\beta$-globin locus. Mol Cell 27: 573-584.

Du M, Perry RL, Nowacki NB, Gordon JW, Salma J, Zhao J, Aziz A, Chan J, Siu KW, McDermott JC. 2008. Protein kinase A represses skeletal myogenesis by targeting myocyte enhancer factor 2D. Mol Cell Biol 28: 2952-2970.

The ENCODE Project Consortium, Dunham I, Kundaje A, Aldred SF, Collins PJ, Davis CA, Doyle F, Epstein CB, Frietze S, Harrow J, et al. 2012. An integrated encyclopedia of DNA elements in the human genome. Nature 489: 57-74.

Fong AP, Yao Z, Zhong JW, Cao Y, Ruzzo WL, Gentleman RC, Tapscott SJ. 2012. Genetic and epigenetic determinants of neurogenesis and myogenesis. Dev Cell 22: 721-735.

Gabut M, Samavarchi-Tehrani P, Wang X, Slobodeniuc V, O'Hanlon D, Sung HK, Alvarez M, Talukder S, Pan Q, Mazzoni EO, et al. 2011. An alternative splicing switch regulates embryonic stem cell pluripotency and reprogramming. Cell 147: 132-146.

Haberland M, Arnold MA, McAnally J, Phan D, Kim Y, Olson EN. 2007. Regulation of HDAC9 gene expression by MEF2 establishes a negative-feedback loop in the transcriptional circuitry of muscle differentiation. Mol Cell Biol 27: 518525.

Hinits Y, Hughes SM. 2007. Mef2s are required for thick filament formation in nascent muscle fibres. Development 134: 25112519.

Iglesias-Bartolome R, Gutkind JS. 2011. Signaling circuitries controlling stem cell fate: To be or not to be. Curr Opin Cell Biol 23: 716-723.

Kalsotra A, Cooper TA. 2011. Functional consequences of developmentally regulated alternative splicing. Nat Rev Genet 12: 715-729.

Khokhlatchev A, Xu S, English J, Wu P, Schaefer E, Cobb MH. 1997. Reconstitution of mitogen-activated protein kinase phosphorylation cascades in bacteria. Efficient synthesis of active protein kinases. J Biol Chem 272: 11057-11062.

Langmead B, Salzberg SL. 2012. Fast gapped-read alignment with Bowtie 2. Nat Methods 9: 357-359.

Liu QC, Zha XH, Faralli H, Yin H, Louis-Jeune C, Perdiguero E, Pranckeviciene E, Munoz-Canoves P, Rudnicki MA, Brand M et al. 2012. Comparative expression profiling identifies differential roles for myogenin and p38 $\alpha$ MAPK signaling in myogenesis. J Mol Cell Biol 4: 386-397.

Martin JF, Miano JM, Hustad CM, Copeland NG, Jenkins NA, Olson EN. 1994. A Mef2 gene that generates a muscle-specific 
isoform via alternative mRNA splicing. Mol Cell Biol 14: 1647-1656.

McNally JG, Muller WG, Walker D, Wolford R, Hager GL. 2000. The glucocorticoid receptor: Rapid exchange with regulatory sites in living cells. Science 287: 1262-1265.

Mukai A, Hashimoto N. 2008. Localized cyclic AMP-dependent protein kinase activity is required for myogenic cell fusion. Exp Cell Res 314: 387-397.

Ohkawa Y, Marfella CG, Imbalzano AN. 2006. Skeletal muscle specification by myogenin and Mef2D via the SWI/SNF ATPase Brg1. EMBO J 25: 490-501.

Ohkawa Y, Yoshimura S, Higashi C, Marfella CG, Dacwag CS, Tachibana T, Imbalzano AN. 2007. Myogenin and the SWI/ SNF ATPase Brg1 maintain myogenic gene expression at different stages of skeletal myogenesis. J Biol Chem 282: 65646570.

Palii CG, Perez-Iratxeta C, Yao Z, Cao Y, Dai F, Davison J, Atkins H, Allan D, Dilworth FJ, Gentleman R, et al. 2011. Differential genomic targeting of the transcription factor TAL1 in alternate haematopoietic lineages. EMBO $J$ 30: 494-509.

Penn BH, Bergstrom DA, Dilworth FJ, Bengal E, Tapscott SJ. 2004. A MyoD-generated feed forward circuit temporally patterns gene expression during skeletal muscle differentiation. Genes Dev 18: 2348-2353.

Potthoff MJ, Olson EN. 2007. MEF2: A central regulator of diverse developmental programs. Development 134: 41314140.

Potthoff MJ, Wu H, Arnold MA, Shelton JM, Backs J, McAnally J, Richardson JA, Bassel-Duby R, Olson EN. 2007. Histone deacetylase degradation and MEF2 activation promote the formation of slow-twitch myofibers. J Clin Invest 117: 2459_ 2467.

Rampalli S, Li L, Mak E, Ge K, Brand M, Tapscott SJ, Dilworth FJ. 2007. p38 MAPK signaling regulates recruitment of Ash2Lcontaining methyltransferase complexes to specific genes during differentiation. Nat Struct Mol Biol 14: 1150-1156.

Reed HC, Hoare T, Thomsen S, Weaver TA, White RA, Akam M, Alonso CR. 2010. Alternative splicing modulates Ubx protein function in Drosophila melanogaster. Genetics 184: 745-758.

Seenundun S, Rampalli S, Liu QC, Aziz A, Palii C, Hong S, Blais A, Brand M, Ge K, Dilworth FJ. 2010. UTX mediates demethylation of $\mathrm{H} 3 \mathrm{~K} 27 \mathrm{me} 3$ at muscle-specific genes during myogenesis. EMBO J 29: 1401-1411.

Shevchenko A, Tomas H, Havlis J, Olsen JV, Mann M. 2006. In-gel digestion for mass spectrometric characterization of proteins and proteomes. Nat Protoc 1: 2856-2860.

Stewart R, Flechner L, Montminy M, Berdeaux R. 2011. CREB is activated by muscle injury and promotes muscle regeneration. PLOS ONE 6: e24714.

Trapnell C, Pachter L, Salzberg SL. 2009. TopHat: Discovering splice junctions with RNA-Seq. Bioinformatics 25: 11051111.

Trapnell C, Williams BA, Pertea G, Mortazavi A, Kwan G, van Baren MJ, Salzberg SL, Wold BJ, Pachter L. 2010. Transcript assembly and quantification by RNA-Seq reveals unannotated transcripts and isoform switching during cell differentiation. Nat Biotechnol 28: 511-515.

Trapnell C, Roberts A, Goff L, Pertea G, Kim D, Kelley DR, Pimentel H, Salzberg SL, Rinn JL, Pachter L. 2012. Differential gene and transcript expression analysis of RNA-seq experiments with TopHat and Cufflinks. Nat Protoc 7: 562-578.

Voss TC, Schiltz RL, Sung MH, Yen PM, Stamatoyannopoulos JA, Biddie SC, Johnson TA, Miranda TB, John S, Hager GL. 2011. Dynamic exchange at regulatory elements during chro- matin remodeling underlies assisted loading mechanism. Cell 146: 544-554.

Wang ET, Sandberg R, Luo S, Khrebtukova I, Zhang L, Mayr C, Kingsmore SF, Schroth GP, Burge CB. 2008. Alternative isoform regulation in human tissue transcriptomes. Nature 456: 470-476.

Wang Z, Zang C, Cui K, Schones DE, Barski A, Peng W, Zhao K. 2009. Genome-wide mapping of HATs and HDACs reveals distinct functions in active and inactive genes. Cell 138: 1019-1031.

Weil PA, Luse DS, Segall J, Roeder RG. 1979. Selective and accurate initiation of transcription at the Ad2 major late promotor in a soluble system dependent on purified RNA polymerase II and DNA. Cell 18: 469-484.

Yaffe D, Saxel O. 1977. Serial passaging and differentiation of myogenic cells isolated from dystrophic mouse muscle. Nature 270: 725-727. 


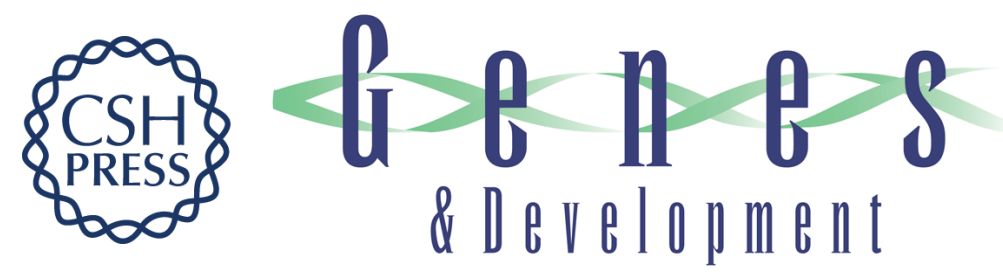

\section{Tissue-specific splicing of a ubiquitously expressed transcription factor is essential for muscle differentiation}

Soji Sebastian, Hervé Faralli, Zizhen Yao, et al.

Genes Dev. 2013, 27: originally published online May 30, 2013

Access the most recent version at doi:10.1101/gad.215400.113

\section{Supplemental http://genesdev.cshlp.org/content/suppl/2013/05/29/gad.215400.113.DC1 Material}

References This article cites 42 articles, 12 of which can be accessed free at: http://genesdev.cshlp.org/content/27/11/1247.full.html\#ref-list-1

License Freely available online through the Genes \& Development Open Access option.
Email Alerting Service

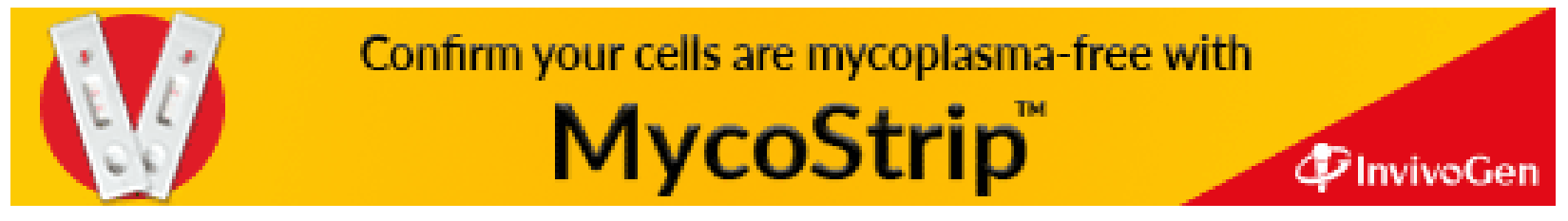

